\title{
The structural and convergent validity of three commonly used measures of self-management in persons with neurological conditions
}

\author{
George Kephart ${ }^{1}$ [D $\cdot$ Tanya L. Packer ${ }^{2} \cdot$ Åsa Audulv ${ }^{3} \cdot$ Grace Warner $^{2}$
}

Accepted: 25 October 2018 / Published online: 2 November 2018

(c) Springer Nature Switzerland AG 2018

\begin{abstract}
Purpose Self-management ability is commonly assessed in chronic disease research and clinical practice. The purpose of this study was to assess the structural and convergent validity of three commonly used self-management outcome measures in a sample of persons with neurological conditions.

Methods We used data from a Canadian survey of persons with neurological conditions, which included three commonly used self-management measures: the Partners in Health Scale (PIH), the Patient Activation Measure (PAM), and the SelfEfficacy for Managing a Chronic Disease Scale (SEMCD). Confirmatory factor analysis was used to assess the structural and convergent validity of the three measures.

Results When treated as single-factor constructs, none of the measurement models provided a good fit to the data. A fourdomain version of the PIH was the best fitting model. Confirmatory factor analysis suggests that the three tools measure different, but correlated constructs.

Conclusions While the PAM, PIH and SEMCD scales are all used as measures of patient self-management, our study indicates that they measure different, but correlated latent variables. None, when treated as single, uni-dimensional construct, provides an acceptable fit to our data. This is probably because self-management is multi-dimensional, as is consistently shown by qualitative evidence. While these measures may provide reliable summative measures, multi-dimensional scales are needed for clinical use and more detailed research on self-management.
\end{abstract}

Keywords Self-management $\cdot$ Self-care $\cdot$ Patient activation $\cdot$ Self-efficacy $\cdot$ Confirmatory factor analysis $\cdot$ Construct validity $\cdot$ Structural validity $\cdot$ Convergent validity

Chronic condition self-management has become a major international focus of health services policy and research. Over 7000 articles with the term "self-management" in the title were found in a PubMed search of the last 5 years alone. Despite this proliferation of research, answers to questions such as "what ingredients are critical to successful outcomes?" and "what works for whom under what conditions?" still elude answers [1-4]. Deficiencies in the

George Kephart

George.kephart@dal.ca

1 Department of Community Health and Epidemiology, Faculty of Medicine, Dalhousie University, 5790 University Avenue, Halifax, NS B3H 1V7, Canada

2 School of Occupational Therapy, Dalhousie University, PO Box 15000, Halifax, NS B3H 4R2, Canada

3 Department of Nursing, Mid-Sweden University, 85170 Sundsvall, Sweden conceptualization and measurement of self-management have been identified as key to this problem [5-8].

The growth in self-management interventions and associated research emerged to fill a gap in service delivery identified by the landmark report 'Crossing the Quality Chasm' [9]. Arising more from practice rather than theoretical foundations, self-management has been defined in different ways, with no single agreed upon definition. A review using concept mapping methodology found two broad conceptualizations [10]. The first defines self-management as behaviors to control disease symptoms, complications and progression. The second, broader definition focuses on behaviors performed to increase overall health and live well with a disease or condition. The later definition is consistent with one of the most commonly cited definitions of self-management proposed by the Institute of Medicine: "tasks that individuals must undertake to live well with one or more chronic conditions. These tasks include having the confidence to 
deal with medical management, role management and emotional management of their conditions" [9]. The practicebased genesis of self-management likely also explains the variety of theoretical foundations cited in the development of interventions and measurement tools. In a recent review of 28 self-management measures, eight reported no specific theoretical foundation, ten reported Bandura's social cognitive theory as their foundation, while the rest provided a wide variety of other theoretical foundations [8].

While disease-specific measures are more numerous, generic self-management measures are widely used [8]. Three of the most commonly used generic measures are the Patient Activation Measure (PAM) [11, 12], the Partners in Health Scale (PIH) [13, 14], and the Self-Efficacy for Managing Chronic Disease Scale (SEMCD) [15]. All three measures are currently used in research and clinical practice settings to measure self-management using a single aggregate score. The SEMCD, the oldest of the three, was specifically designed to measure effectiveness of the Chronic Disease Self-Management Program (CDSMP), a patient education program underpinned by Social Cognitive Theory [16]. While developers of the PAM and the PIH both cite the SEMCD and social cognitive theory as influential in their development, neither is based on a specific conceptual model. While the PAM was developed to measure "patient activation", a term introduced in the Chronic Care Model [17-19], the concept is not clearly defined. Both the PAM and PIH employed similar reviews of literature and expert clinical opinion as the basis for model development.

Since all three measures are used in the research literature to measure chronic disease "self-management", and the conceptual basis for differentiating the three measures is unclear, it is important to assess their structural and convergent validity. However, there have been no direct comparisons of these measures in the literature, as they have not been collected in the same sample. The Everyday Experience of Living with and Managing the Impact of a Neurological Condition (LINC) study [20], which collected data on the PAM, PIH, and SEMCD in a diverse sample of Canadian adults with neurological conditions, provides a unique opportunity to assess the structural and convergent validity of these three measures. Persons with neurological conditions provide a diverse population for this assessment. Neurological conditions are characterized by a broad range of symptoms, disease trajectories, and a range of self-management tasks [21]. Compared to some chronic conditions (diabetes, cardiovascular disease) neurological conditions often result in greater restrictions and disability, earlier in the trajectory of the disease. As with the general population, persons with neurologic conditions also have other common, non-neurological chronic conditions.

The purpose of this paper is to assess in a large sample Canadian adults with neurological conditions, the structural and convergent validity of the PAM, PIH and SEMCD. To assess structural validity, we used confirmatory factor analysis to assess whether the observed data fit the hypothesized single-construct model implied by each measure. To assess convergent validity, we used confirmatory factor analysis to estimate the correlations between the latent constructs, and to assess whether items from the three scales measure the same latent construct, or three different latent constructs.

\section{Methods}

\section{Data}

Data for this study came from the LINC study [20], which was a component of the National Population Study on Neurological Conditions, an initiative to understand and plan for the long term needs of people living with neurological conditions in Canada [22]. The LINC study was a mixed methods study which included a cross-sectional pan-Canadian survey $(n=787)$, a nested cohort study $(n=125)$ and a nested qualitative multiple-perspective study $(n=15)$. Data for this paper were extracted from the cross-sectional survey, which included adults aged 17 and over living with at least one neurological condition. Participants with 14 specific conditions were the primary focus, but participants with any neurological condition were included. They were largely recruited through a coalition of charitable organizations, the Neurological Health Charities of Canada, but additional strategies (non-government agencies, registries, word-of-mouth) were also employed [20]. Data were collected between September 14, 2011 and July 1, 2012 via a voluntary, online survey; however, to accommodate preference and ability, telephone as well as pencil and paper options were available. Ethics approval for the LINC study was obtained from research ethics review boards at Health Canada and three university ethics review boards.

Subjects for this study were respondents with valid total scores, as per tool coding protocols, for all three tools $(N=742)$. Across the 31 items comprising the three measures, $26 \%$ of subjects had item non-response or "not applicable" responses on at least one item. However, missing data on single items were rare (Appendix Tables 9, 10 11), most subjects had missing data on only one or two items across the three scales, and no pattern of missing data comprised more than $4 \%$ of the sample. Having a "not applicable" response on a medication item in the PAM was the most common pattern of missing data $(4 \%)$. To enable use of data for all 742 subjects in the analysis, multivariate imputation of missing data using chained ordinal logistic regression equations was used, employing items from all three measures as predictors [23]. This is preferable to the handling of missing data using maximum likelihood estimation of model 
parameters, which assumes joint normality of all variables and that the data are missing at random after accounting for only the variables in each model (which differ from model to model in our analyses). Our method thus makes less restrictive missing data assumptions which are consistent across all models we estimate. Three sets of imputed values for the missing data were computed with different random number seeds [24]. Models were estimated with each of the three imputed data sets and compared. All parameter estimates and fit statistics were nearly identical, demonstrating minimal variation introduced by the imputation procedure. Reported results are from the first set of imputed data.

\section{Measures}

The SEMCD scale was developed to assess outcomes of the Stanford Chronic Disease Self-Management Program (CDSMP), which aimed to enhance patients' self-efficacy for chronic disease self-management $[15,25]$. The current measure is built from six of ten items from the original Selfefficacy to Manage Symptoms and the Self-efficacy to Manage Disease scales (2 of 10 scales designed to evaluate the Stanford program) [25]. Despite extensive use, little information is available on how the original scales were designed or items selected. A German, Persian, and an international validation study of both the English and Spanish versions all show the SEMCD to be uni-dimensional and internally consistent [25-27].

The PIH has progressed through two revisions. In this study the most recent version was used [14]. Development of the PIH was motivated by the lack of a generic measure of self-management that could be used to assess patient selfmanagement knowledge, skill, and ability [13]. Drawing on a literature review, they extracted core concepts and created items to measure different aspects of chronic condition selfmanagement. Revisions added items to assess empowerment and the impact of the condition on physical activities, emotions, and social life $[14,28]$. The current version of the PIH includes 12 items, scored on a 0 to 8 response scale [14]. The scoring instructions and clinical application provide a single total score; however, psychometric analysis found that the PIH consists of four interrelated factors (knowledge, partnership in treatment, recognition and management of symptoms, and coping) conceptualized as part of a single, higher order latent variable [14].

The PAM was designed to measure the concept of "patient activation", and to fill the need for a tool that "includes a broad range of elements involved in activation, including the knowledge, skills, beliefs and behaviors that patients need to manage a chronic illness" [11]. In 2005 an original 22 item scale was reduced to the 13-item short form used in this study [12]. The scale was developed using Rasch methods to create a uni-dimensional, interval-level scale.
Items are sequenced and scaled by "difficulty" of activation, with higher scores indicating greater activation. The PAM has been translated and validated in many countries [29-34], and across multiple populations including mental health [35, 36], elective lumbar spine surgery [37], multi-morbid older adults [37], hospitalized patients [38], and rural populations [39]. Overall these studies provide evidence that the PAM is uni-dimensional, with good reliability and construct validity. There is, however, evidence of variation in differential item scaling across populations [32, 40].

\section{Analytic approach}

Confirmatory factor analysis (CFA) was the primary analytic tool used to assess the structural and convergent validity of the three tools. CFA is well suited for testing a measurement theory against observed data by examining overall fit and specific areas in which the model does not fit [41]. To facilitate comparisons with published literature, Chronbach's Alpha was computed for each of the scales as a lower bound estimate of reliability. Alpha is not a suitable measure of structural validity or uni-dimensionality of a scale $[42,43]$.

All CFA models specified uncorrelated errors between indicators and were identified both by specifying the first item as the reference indicator for each latent variable (unstandardized solutions) and by fixing the variance of the latent variable to 1.0 (standardized solutions). We report standardized results as they facilitate comparative performance of indicators.

Non-normality of the data is a concern for model estimation. Both PAM and PIH items were skewed, and the PAM included just four response categories ranging from strongly disagree to strongly agree, with only a small percentage of respondents selecting the strongly disagree category (see Appendix Tables 9, 10 11). Accordingly, robust maximum likelihood estimation was employed with the Satorra-Bentler method to generate Chi-square fit statistics, associated fit indices, and standard errors [44]. This method has been shown to be robust to non-normality, and preferable to alternative estimation methods for non-normal data [45]. As a validation check, comparison models were also estimated using general linear models with an ordinal logistic link function. Commonly used fit indices and diagnostics are not available for this method, but it produced comparable factor loadings and correlations between latent variables to those obtained by robust maximum likelihood estimates. We thus report the maximum likelihood estimates. All data were analyzed using STATA 15 [24].

To assess the structural validity of each model, separate, single-factor CFA models for each of the three measures were estimated. For the PIH, we also estimated a model for its hypothesized four-factor structure, assuming correlated latent variables [14]. Structural validity was assessed 
by examining model fit using the Chi-Square goodness of fit statistic, the root mean squared error of approximation (RMSEA), the Comparative Fit Index (CFI), and the TuckerLewis Index (TLI). Criteria for overall good fit of a model were an RMSEA close to or below 0.06, and CFI and TLI results close to or greater than 0.95 [46]. Standardized factor loadings were examined to assess and compare the association of latent variables with individual items. To identify localized areas of poor fit within the models, and possible reasons for poor fit, standardized covariance residuals and modification indices were examined. As our objective was to assess structural validity, and not to refine the measures, we did not revise the models to improve model fit.

Convergent validity was assessed in two ways. First, we estimated a three-factor model incorporating all three measurement tools as latent, correlated variables (Fig. 1). Estimated correlations between the three models were used to assess the degree of convergent validity between the measures. Second, we compared the fit of a three-factor model to a reduced, single-factor model that treated the items of all three scales as indicators of a single latent variable. A significant difference in fit of the models (using a Chi-square difference test) and large difference in global indices of model fit was considered evidence that the three scales do not measure the same latent construct.

\section{Results}

Table 1 shows characteristics of the study sample. Subjects had a range of neurological conditions, with multiple sclerosis, Parkinson's disease, and brain injury being the most common. Compared to the general population of persons with chronic conditions, the sample is younger, more likely to be female, and has higher socio-economic status. Appendix Tables 9, 1011 show detailed distributions of responses to the items of each scale.

Our results show weaknesses in the structural validity of all three measures. CFA models for each of the three measures, treated as single-factor measures, do not meet the criteria for good overall fit (Table 2). This is particularly the case for the PIH and the PAM models, which have large Chi-square values, RMSEA values significantly larger than 0.05 , and CFI and TLI values well below the 0.95 criteria.
Fig. 1 Three-factor confirmatory factor analysis model for the PAM, PIH, and SEMCD

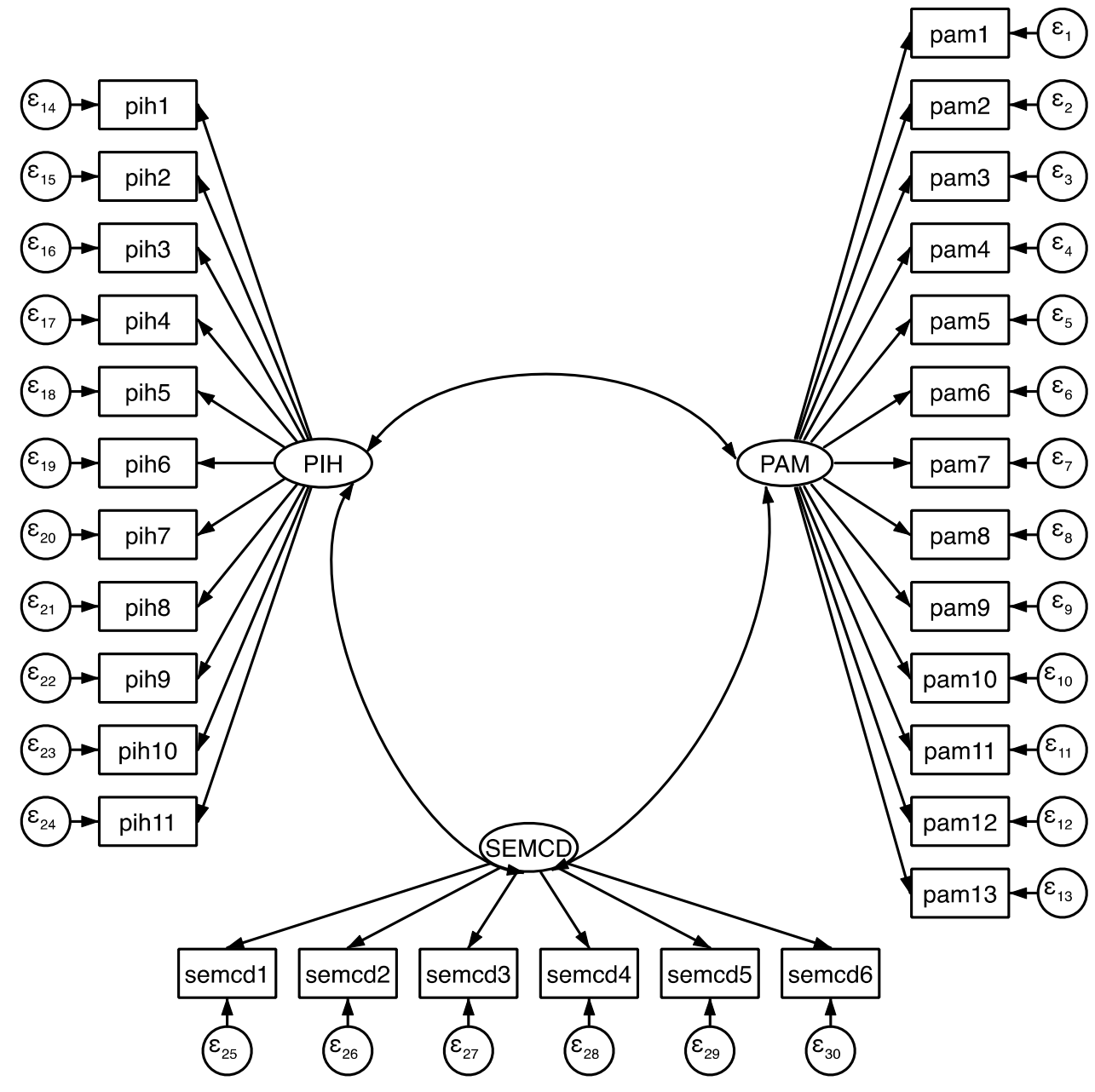


Table 1 Characteristics of the study sample

\begin{tabular}{|c|c|c|}
\hline & $N$ & Percent \\
\hline \multicolumn{3}{|l|}{ Age group } \\
\hline$\leq 25$ & 38 & 5.1 \\
\hline $26-35$ & 97 & 13.1 \\
\hline $36-45$ & 105 & 14.2 \\
\hline $46-55$ & 191 & 24.4 \\
\hline $56-65$ & 181 & 17.0 \\
\hline $66-75$ & 95 & 12.9 \\
\hline$>75$ & 31 & 4.2 \\
\hline \multicolumn{3}{|l|}{ Sex } \\
\hline Male & 261 & 35.2 \\
\hline Female & 477 & 64.3 \\
\hline \multicolumn{3}{|l|}{ Marital status } \\
\hline Married/common-law & 459 & 61.9 \\
\hline Widowed/separated/divorced & 119 & 16.1 \\
\hline Single, never married & 160 & 21.6 \\
\hline \multicolumn{3}{|l|}{ Education level } \\
\hline$<$ Secondary & 50 & 7.1 \\
\hline Secondary graduate & 88 & 12.0 \\
\hline Some post-secondary & 56 & 8.1 \\
\hline Post-secondary degree & 517 & 72.8 \\
\hline Missing & 31 & 4.2 \\
\hline \multicolumn{3}{|l|}{ Household income } \\
\hline$<\$ 20,000$ & 88 & 11.9 \\
\hline$\$ 20,000-\$ 59,999$ & 201 & 27.1 \\
\hline$\$ 60,000-\$ 89,999$ & 108 & 14.1 \\
\hline$\$ 90,000+$ & 126 & 17.0 \\
\hline Missing & 219 & 30.0 \\
\hline \multicolumn{3}{|c|}{ Most common neurological conditions in sample } \\
\hline Multiple sclerosis & 172 & 23.4 \\
\hline Parkinson's disease & 148 & 20.1 \\
\hline Brain injury & 105 & 14.6 \\
\hline Epilepsy & 98 & 13.3 \\
\hline Neuromuscular diseases & 91 & 12.3 \\
\hline
\end{tabular}

The SEMCD provides a better fit to the data than the PIH and PAM. While the CFI and TLI for the SEMCD model approach the criteria for good model fit, the RMSEA is considerably higher than the 0.05 criteria.

Clues to the reasons for poor fit are provided by the factor loadings in Tables 3, 4, 5 and 6. Squared, standardized loadings measure the correlation between the items and their latent constructs. Factor loadings exceeding 0.8 (a correlation of 0.64 with the latent construct) are desirable for good model fit [41]. In the case of PAM, standardized factor loadings for the items range between 0.45 and 0.75 , indicating weak to moderate correlations (ranging from 0.20 to 0.56 ) with the latent construct. With only four response categories, and very few subjects strongly disagreeing with any of the items, PAM items have low variation. This may contribute to weak model fit. For the single-factor PIH model, standardized loadings are variable in magnitude, with just three items $(9,10$, and 11) exceeding 0.70 . Two of the items (3 and 6) stand out as having very weak loadings. Both items were designed to measure partnership, but their wording suggests that they measure adherence (see Appendix Table 8 for item wordings). Dropping the two items from the model (not shown) did not result in a well-fitting model. The SEMCD loadings exceed 0.80 for 4 of the 6 items in the scale. Loadings for the last two items in the scale are weaker than the first four.

The results suggest that unspecified multidimensionality of self-management ability in the single-factor models may contribute to poor model fit. In each of the three one-factor models, modification indices (not shown) indicate substantial residual correlation of error terms for many pairs of items, and numerous item covariances have high standardized residuals (e.g., 26\% of the PIH, $10 \%$ of the PAM, and $6 \%$ of the SEMCD standardized covariance residuals exceed the commonly used cut-off of \pm 2.58 ). The single-factor PIH model has seven modification indices indicating expected correlated errors greater than 0.30, while the PAM has three and the SEMCD has two. Other, unspecified latent variables are common reasons for correlated errors and high covariance residuals [41]. While correlated errors may also result from "method effects" due to similarly worded items (e.g., the PAM has several similarly worded items), this cannot account for most of the correlated errors identified by the modification indices.

The PIH, treated as four-factor model, provides much better fit to the data than any of the single-factor models (Table 2). The CFI and TLI both met the criteria for good fit, the RMSEA was close to 0.05 (upper bound of the $90 \%$ confidence interval is 0.065 ). Two of the four factors have just two items each, limiting model fit and reliability. Examination of the factor loadings (Table $2 b$ ) shows that the two items included in the patient-provider partnership factor (pih3 and pih6), have weak loadings. Estimated correlations between the four PIH domains are modest, ranging from 0.37 to 0.65 , indicating discriminant validity of the factors (Table 7).

Convergent validity of the three measures is modest. Correlations between latent variables corresponding to the three measurement tools range from 0.63 to 0.76 (Table 7 ). The PAM and PIH are more strongly correlated with each other than they are with the SEMCD. Moreover, a CFA model treating all items as indicators of a single latent variable has significantly poorer fit than a three-factor model specifying the measures as separate, but correlated constructs (see bottom 2 rows of Table 2). All fit indices are substantially poorer for the single-factor model, and a Chi-square difference test indicates significantly better fit for the three-factor model $(p<.001)$. 
Table 2 Chronbach's alphas and indices of confirmatory factor analysis model fit
Table 3 PIH item distributions, and single-factor CFA standardized factor loadings and error variances

\begin{tabular}{llccrr}
\hline Model & $\begin{array}{l}\text { Chronbach's } \\
\text { alpha }^{\mathrm{a}}\end{array}$ & Chi-square (df) $^{\mathrm{c}}$ & RMSEA (95\% CI) $^{\mathrm{c}}$ & CFI $^{\mathrm{c}}$ & TLI $^{\mathrm{c}}$ \\
\hline PIH & 0.847 & $707.1(54)$ & $0.168(0.159,0.176)$ & 0.681 & 0.610 \\
PIH (4 factor) & $\mathrm{b}$ & $99.9(48)$ & $0.056(0.046,0.065)$ & 0.975 & 0.965 \\
PAM & 0.884 & $573.3(65)$ & $0.118(0.111,0.126)$ & 0.820 & 0.784 \\
SEMCD & 0.902 & $106.8(9)$ & $0.168(0.148,0.188)$ & 0.946 & 0.909 \\
Single factor & \\
Three factor $^{\mathrm{d}}$ & 0.931 & $3609.1(434)$ & $0.116(0.113,0.119)$ & 0.632 & 0.606 \\
\hline
\end{tabular}

${ }^{a}$ Chronbach's alpha coefficients were computed from standardized item scores without missing data imputation

${ }^{\mathrm{b}}$ Chronbach's alpha coefficients for the four domains of PIH are: knowledge of illness and treatments $=0.870$, patient-provider partnership $=.610$, symptom management $=0.741$, and coping $=0.850$.

${ }^{\mathrm{c}}$ Satorra-Bentler scaled Chi-square statistics (degrees of freedom) are versus the saturated model, and $p<.001$ for all models. RMSEA is the root mean squared error of approximation (and 90\% CI), CFI is the comparative fit index and TLI is the Tucker-Lewis Index

${ }^{\mathrm{d}}$ Chi-square test of improvement in fit of the three versus single factor models: chi square (df) $=620.0$ (3), $p<.001$

\begin{tabular}{llllll}
\hline Item & \% Missing & Mean & Std. Dev. & Standardized loading $(95 \%$ CI) & Error variance \\
\hline pih1 & 0.40 & 7.77 & 1.54 & $0.497(0.437,0.558)$ & 0.753 \\
pih2 & 0.54 & 7.46 & 1.78 & $0.531(0.469,0.592)$ & 0.719 \\
pih3 & 1.08 & 8.26 & 1.39 & $0.266(0.163,0.370)$ & 0.929 \\
pih4 & 0.27 & 8.14 & 1.44 & $0.477(0.404,0.550)$ & 0.772 \\
pih5 & 0.67 & 7.78 & 1.76 & $0.540(0.469,0.610)$ & 0.709 \\
pih6 & 0.13 & 8.79 & 0.68 & $0.310(0.215,0.405)$ & 0.904 \\
pih7 & 1.08 & 7.44 & 1.85 & $0.585(0.531,0.639)$ & 0.658 \\
pih8 & 1.08 & 7.59 & 1.66 & $0.596(0.536,0.656)$ & 0.645 \\
pih9 & 1.35 & 6.68 & 2.19 & $0.720(0.673,0.766)$ & 0.482 \\
pih10 & 1.89 & 6.50 & 2.13 & $0.769(0.729,0.808)$ & 0.409 \\
pih11 & 0.67 & 6.43 & 2.25 & $0.713(0.667,0.760)$ & 0.491 \\
pih12 & 0.27 & 6.85 & 2.15 & $0.645(0.594,0.695)$ & 0.584
\end{tabular}

\section{Discussion}

In our sample of persons with neurological conditions, none of the three measurement tools, when treated as uni-dimensional models, had high structural validity. Of the three, the SEMCD was the best fitting scale, despite having only six items. Both the PAM and the single-factor PIH had fit statistics that fell far short of standards for a good fitting model. The PAM was developed based on Rasch methods, which raises the question of appropriateness of using confirmatory factor analysis to assess its fit. However, the inclusion of intercepts in the confirmatory factor analysis model captures differences in difficulty of the items and is thus very similar in its specification to the rating scale model on which scaling of the PAM is based [41]. Previously reported validation results for the PAM, using the rating scale model on the same data, also identified problems with fit, such as item difficulties that deviate from the original PAM scoring [40].

There are a number of possible reasons for poor fit of the measurement models, including poorly performing items, lack of applicability of the tools to the study population, or multidimensionality of the underlying constructs. We found limited evidence that poorly performing items account for poor model fit in any of the three scales. Two of 12 items in the PIH had low standardized factor loadings, but elimination of these two items did not result in a well-fitting model. In the PAM and SEMCD no items stood out as especially poor performers. The PAM had relatively weak standardized factor loadings for all items. Lack of variation across the four PAM response categories, and ceiling effects, have been noted in the LINC and other data sources [30, 39, 40, 47]. This may contribute to the poor fit of the PAM. Lack 
Table 4 PIH four-factor model: CFA standardized factor loadings and error, and variances

\begin{tabular}{|c|c|c|c|}
\hline Domain & Item & $\begin{array}{l}\text { Standardized loading (95\% } \\
\text { CI) }\end{array}$ & Error variance \\
\hline \multicolumn{4}{|c|}{ Knowledge of illness and treatments } \\
\hline & pih1 & $0.817(0.771,0.864)$ & 0.332 \\
\hline & pih2 & $0.943(0.890,0.995)$ & 0.111 \\
\hline \multicolumn{4}{|c|}{ Patient-provider partnership } \\
\hline & pih3 & $0.394(0.268,0.519)$ & 0.845 \\
\hline & pih4 & $0.667(0.592,0.742)$ & 0.556 \\
\hline & pih5 & $0.719(0.648,0.790)$ & 0.483 \\
\hline & pih6 & $0.384(0.247,0.521)$ & 0.853 \\
\hline \multicolumn{4}{|c|}{ Symptom management } \\
\hline & pih7 & $0.759(0.707,0.811)$ & 0.424 \\
\hline & pih8 & $0.775(0.705,0.844)$ & 0.400 \\
\hline \multicolumn{4}{|l|}{ Coping } \\
\hline & pih9 & $0.702(0.651,0.752)$ & 0.508 \\
\hline & pih10 & $0.880(0.844,0.916)$ & 0.226 \\
\hline & pih11 & $0.797(0.752,0.842)$ & 0.366 \\
\hline & pih12 & $0.685(0.635,0.734)$ & 0.531 \\
\hline
\end{tabular}

of applicability of the tools to persons with neurologic conditions is possible, but unlikely given the diverse range of symptoms characterizing the included conditions. Moreover, poor fit of a single-factor PIH model has also been found in diverse chronic disease and multi-morbid hospital populations [14, 48].

The most plausible reason for weak structural validity of the models is that self-management is multi-dimensional, not uni-dimensional. There is a strong qualitative evidence for the multidimensionality of self-management. The extensive body of qualitative literature consistently shows that self-management consists of different skills, abilities and attitudes [5, 10, 49-55]. A multi-dimensional version of the $\mathrm{PIH}$ was the best fitting model in our analyses, and this is consistent with analysis of the PIH in another recent study [14]. Moreover, modification indices indicating correlated errors between items for all the single-factor models suggest the presence of other factors.

We found modest evidence for convergent validity of the three measures. The analysis suggests that they measure different, but correlated latent variables. Given the poor fit of the three measurement models individually, our evidence
Table 5 PAM: item distributions and CFA standardized factor loadings and error variances

\begin{tabular}{llllll}
\hline Item & \% Missing & Mean & Std.Dev. & Standardized loading $(95 \%$ CI $)$ & Error variance \\
\hline pam1 & 0.67 & 3.50 & 0.69 & $0.449(0.378,0.519)$ & 0.799 \\
pam2 & 0.67 & 3.50 & 0.65 & $0.494(0.434,0.554)$ & 0.756 \\
pam3 & 1.62 & 3.00 & 0.80 & $0.582(0.526,0.639)$ & 0.661 \\
pam4 & 6.87 & 3.40 & 0.64 & $0.583(0.530,0.636)$ & 0.660 \\
pam5 & 1.48 & 3.24 & 0.70 & $0.646(0.591,0.700)$ & 0.583 \\
pam6 & 1.35 & 3.29 & 0.71 & $0.574(0.512,0.636)$ & 0.671 \\
pam7 & 2.70 & 3.36 & 0.63 & $0.618(0.562,0.673)$ & 0.619 \\
pam8 & 0.94 & 3.18 & 0.75 & $0.613(0.560,0.665)$ & 0.625 \\
pam9 & 3.23 & 3.11 & 0.73 & $0.605(0.547,0.662)$ & 0.634 \\
pam10 & 1.62 & 2.80 & 0.82 & $0.619(0.569,0.669)$ & 0.617 \\
pam11 & 2.43 & 2.91 & 0.69 & $0.707(0.663,0.753)$ & 0.499 \\
pam12 & 1.62 & 2.75 & 0.74 & $0.749(0.708,0.790)$ & 0.440 \\
pam13 & 1.35 & 2.66 & 0.79 & $0.610(0.560,0.661)$ & 0.628 \\
\hline
\end{tabular}

Table 6 SEMCD: item distributions and CFA standardized factor loadings and error variances

\begin{tabular}{llllll}
\hline Item & \% Missing & Mean & Std. Dev. & Standardized loading $(95 \%$ CI $)$ & Error variance \\
\hline semcd1 & 0.00 & 5.42 & 2.80 & $0.807(0.774,0.840)$ & 0.349 \\
semcd2 & 0.67 & 6.05 & 2.75 & $0.812(0.773,0.850)$ & 0.341 \\
semcd3 & 0.40 & 6.22 & 2.75 & $0.817(0.780,0.853)$ & 0.333 \\
semcd4 & 0.54 & 6.02 & 2.65 & $0.836(0.799,0.873)$ & 0.302 \\
semcd5 & 1.08 & 6.68 & 2.56 & $0.705(0.654,0.755)$ & 0.504 \\
semcd6 & 0.40 & 6.84 & 2.68 & $0.679(0.631,0.728)$ & 0.539 \\
\hline
\end{tabular}


Table 7 Estimated correlations between latent variables from confirmatory factor analysis models

\begin{tabular}{|c|c|c|}
\hline & Correlation & $95 \% \mathrm{CI}$ \\
\hline \multicolumn{3}{|l|}{ Between Scales $^{\mathrm{a}}$} \\
\hline PIH, PAM & 0.757 & $(0.718,0.797)$ \\
\hline PIH, SEMCD & 0.722 & $(0.677,0.767)$ \\
\hline PAM, SEMCD & 0.626 & $(0.574,0.677)$ \\
\hline \multicolumn{3}{|l|}{ Between PIH domains $^{\mathrm{b}}$} \\
\hline Knowledge, partnership & 0.646 & $(0.556,0.737)$ \\
\hline Knowledge, symptom manage & 0.433 & $(0.346,0.520)$ \\
\hline Knowledge, coping & 0.368 & $(0.293,0.443)$ \\
\hline Partnership, symptom manage & 0.618 & $(0.520,0.715)$ \\
\hline Partnership, coping & 0.521 & $(0.436,0.605)$ \\
\hline Symptom manage, coping & 0.623 & $(0.556,0.691)$ \\
\hline
\end{tabular}

${ }^{a}$ Estimated from a three-factor model treating PIH as a single factor ${ }^{b}$ Estimated from a PIH model treating the four domains as separate factors

for this conclusion should be treated with some caution. Estimates of correlation between poorly fitting latent variables may not be robust [41]. One explanation for differentiation of the scales is that they emphasize, to a greater or lesser degree, different aspects of self-management identified in the qualitative literature. For example, PAM does not include items directly measuring management of social and emotional aspects of self-management, while the other two scales do. Self-efficacy, measured by the SEMCD, may be considered a precondition for the acquisition and execution of more specific self-management strategies, such as symptom monitoring or life-style changes, which are measured by the PIH and PAM.

What do these results mean for the utility of the three measures? Evidence from many studies shows each of the three tools provides reliable measures of self-management ability, with evidence of construct validity $[25,26,28,35$, $38,56-59]$. Poor fit of the models in our study does not contradict these findings. Rather, it suggests these measures should be understood as composite scales of self-management, with each scale summarizing unidentified and somewhat different dimensions of self-management.

Measures that are multi-dimensional, or that examine more narrow aspects of self-management, may have greater clinical utility and enhance the depth of research. There are many alternatives to the three measures of self-management examined in this study, and researchers and practitioners should give careful consideration to the measure that is most appropriate for the purpose. A recent scoping review, identified 28 diverse self-management measures, of which 13 were multi-dimensional, and 20 were condition specific [8]. They were based on a variety of definitions of self-management, grounded in a variety of theoretical perspectives, and the multi-dimensional measures were inconsistent in their domain definitions. The review highlighted the need for measurement of self-management to catch up with the evidence on self-management as a multifaceted, ongoing process. Measures are needed which move beyond a composite assessment to differentiate patients' self-management strengths and need for support, and that can be used in research to investigate the critical ingredients of self-management interventions for patients with different self-management needs.

Funding The funding was provided by Public Health Agency of Canada, Nova Scotia Health Research Foundation (Grant No. PSODI-2015-10083), Institute of Health Services and Policy Research.

\section{Appendix}

See Tables 8, 9, 10 and 11 . 
Table 8 Item wordings for the PAM, PIH and SEMCD

PAM

pam 1 When all is said and done, I am the person who is responsible for taking care of my health

pam 2 Taking an active role in my own health care is the most important thing that affects my health

pam 3 I am confident I can help prevent or reduce problems associated with my health

pam 4 I know what each of my prescribed medications do

pam 5 I am confident that I can tell whether I need to go to the doctor or whether I can take care of a health problem myself

pam 6 I am confident that I can tell a doctor concerns I have even when he or she does not ask

pam 7 I am confident that I can follow through on medical treatments I may need to do at home

pam 8 I understand my health problems and what causes them

pam 9 I know what treatments are available for my health problems

pam 10 I have been able to maintain (keep up with) life-style changes, like eating right or exercising

pam 11 I know how to prevent problems with my health

pam 12 I am confident I can figure out solutions when new problems arise with my health

pam 13 I am confident that I can maintain life-style changes, like eating right and exercising, even during times of stress

Partners in Health Scale (PIH)

pih 1 Overall, what I know about my health condition(s) is

pih 2 Overall, what I know about my treatment, including medications for my health condition(s) is

pih 3 I take medications or carry out the treatments asked by my doctor/health worker

pih 4 I share in decisions made about my health condition(s) with my doctor or health worker

pih 5 I am able to deal with health professionals to get the services I need that fit with my culture, values and beliefs

pih 6 I attend appointments as asked by my doctor or health worker

pih 7 I keep track of my symptoms and early warning signs 9 (e.g., blood sugar levels, peak flow, weight, shortness of breath, pain, sleep problems, mood)

pih 8 I take action when my early warning signs and symptoms get worse

pih 9 I manage the effect of my health conditions(s) on my physical activity (i.e., walking, household tasks)

pih 10 I manage the effect of my health conditions(s) on how I feel (i.e., my emotions and spiritual wellbeing)

pih 11 I manage the effect of my health condition(s) on my social life (i.e., how I mix with other people)

pih12 Overall, I manage to live a health life (e.g., no smoking, moderate alcohol, health food, regular physical activity, manage stress)

SEMCD

semcd1 How confident are you that you can keep the fatigue caused by your disease from interfering with the things you want to do?

semcd2 How confident are you that you can keep the physical discomfort or pain of your disease from interfering with the things you want to do?

semcd3 How confident are you that you can keep the emotional distress caused by your disease from interfering with the things you want to do?

semcd4 How confident are you that you can keep any other symptoms or health problems you have from interfering with the things you want to do?

semcd5 How confident are you that you can do the different tasks and activities needed to manage your health condition so as to reduce your need to see a doctor?

semcd6 How confident are you that you can do things other than just taking medication to reduce how much your illness affects your everyday life?

Table 9 Distribution of PIH Item Scores

\begin{tabular}{|c|c|c|c|c|c|c|c|c|c|c|c|c|}
\hline & pih1 (\%) & pih2 $(\%)$ & pih3 (\%) & pih4 $(\%)$ & pih5 (\%) & pih6 (\%) & pih7 (\%) & pih8 (\%) & pih9 $(\%)$ & pih10 $(\%)$ & pih11 (\%) & pih12 (\%) \\
\hline 1 & 0.8 & 1.8 & 1.5 & 0.3 & 1.5 & 0.0 & 1.5 & 0.5 & 3.9 & 3.1 & 4.3 & 3.4 \\
\hline 2 & 0.5 & 0.9 & 0.0 & 0.5 & 0.8 & 0.1 & 0.7 & 0.5 & 1.2 & 2.3 & 2.3 & 1.5 \\
\hline 3 & 0.5 & 1.4 & 0.3 & 0.4 & 1.2 & 0.3 & 2.6 & 1.6 & 3.4 & 4.2 & 3.8 & 3.8 \\
\hline 4 & 1.1 & 1.6 & 0.4 & 0.8 & 1.9 & 0.0 & 1.2 & 1.6 & 3.9 & 4.7 & 7.0 & 4.6 \\
\hline 5 & 7.0 & 9.3 & 3.6 & 7.0 & 8.1 & 0.9 & 12.9 & 11.1 & 21.3 & 21.3 & 20.8 & 15.5 \\
\hline 6 & 7.1 & 6.7 & 1.8 & 3.0 & 4.2 & 0.1 & 5.8 & 5.8 & 4.5 & 5.9 & 5.8 & 6.1 \\
\hline 7 & 15.5 & 17.3 & 9.4 & 9.0 & 10.1 & 1.6 & 11.6 & 14.0 & 15.1 & 16.3 & 13.5 & 16.0 \\
\hline 8 & 23.5 & 24.4 & 19.1 & 17.3 & 21.8 & 11.2 & 23.5 & 23.6 & 17.8 & 19.7 & 19.5 & 20.0 \\
\hline 9 & 43.5 & 36.1 & 62.8 & 61.5 & 49.7 & 85.6 & 39.2 & 40.2 & 27.6 & 20.6 & 22.4 & 29.0 \\
\hline Missing & 0.4 & 0.5 & 1.1 & 0.3 & 0.7 & 0.1 & 1.1 & 1.1 & 1.4 & 1.9 & 0.7 & 0.3 \\
\hline
\end{tabular}


Table 10 Distribution of PAM Item Scores

\begin{tabular}{|c|c|c|c|c|c|c|c|c|c|c|c|c|c|}
\hline & $\begin{array}{l}\text { pam1 } \\
(\%)\end{array}$ & $\begin{array}{l}\text { pam2 } \\
(\%)\end{array}$ & $\begin{array}{l}\text { pam3 } \\
(\%)\end{array}$ & $\begin{array}{l}\text { pam4 } \\
(\%)\end{array}$ & $\begin{array}{l}\text { pam5 } \\
(\%)\end{array}$ & $\begin{array}{l}\text { pam6 } \\
(\%)\end{array}$ & $\begin{array}{l}\text { pam7 } \\
(\%)\end{array}$ & $\begin{array}{l}\text { pam8 } \\
(\%)\end{array}$ & $\begin{array}{l}\text { pam9 } \\
(\%)\end{array}$ & $\begin{array}{l}\text { pam10 } \\
(\%)\end{array}$ & $\begin{array}{l}\text { pam11 } \\
(\%)\end{array}$ & $\begin{array}{l}\text { pam12 } \\
(\%)\end{array}$ & $\begin{array}{l}\text { pam13 } \\
(\%)\end{array}$ \\
\hline 1 & 2.6 & 1.4 & 3.6 & 0.4 & 1.6 & 1.9 & 0.4 & 2.8 & 2.6 & 6.3 & 2.7 & 4.0 & 6.2 \\
\hline 2 & 3.8 & 4.6 & 20.5 & 6.5 & 10.2 & 9.3 & 6.7 & 12.4 & 13.6 & 26.2 & 20.0 & 29.7 & 34.5 \\
\hline 3 & 34.8 & 36.7 & 46.9 & 41.8 & 49.2 & 45.6 & 47.7 & 48.0 & 51.4 & 47.0 & 58.1 & 51.4 & 44.6 \\
\hline 4 & 58.2 & 56.7 & 27.4 & 44.5 & 37.5 & 41.9 & 42.5 & 35.9 & 29.3 & 18.9 & 16.9 & 13.3 & 13.3 \\
\hline Missing & 0.7 & 0.7 & 1.6 & 6.9 & 1.5 & 1.4 & 2.7 & 0.9 & 3.2 & 1.6 & 2.4 & 1.6 & 1.4 \\
\hline
\end{tabular}

Table 11 Distribution of SEMCD Item Scores

\begin{tabular}{lcccccc}
\hline & semcd1 $(\%)$ & semcd2 $(\%)$ & semcd3 $(\%)$ & semcd4 $(\%)$ & semcd5 $(\%)$ & semcd6 $(\%)$ \\
\hline 1 & 12.8 & 8.0 & 7.3 & 8.6 & 5.5 & 7.1 \\
2 & 7.4 & 6.9 & 5.8 & 4.6 & 3.9 & 2.7 \\
3 & 10.7 & 7.8 & 7.7 & 6.9 & 4.9 & 4.3 \\
4 & 7.7 & 6.6 & 6.2 & 7.3 & 4.9 & 6.2 \\
5 & 8.6 & 8.9 & 11.3 & 12.7 & 10.5 & 7.1 \\
6 & 9.2 & 11.2 & 8.8 & 9.7 & 9.2 & 8.8 \\
7 & 15.9 & 14.4 & 12.8 & 14.7 & 13.2 & 12.1 \\
8 & 14.3 & 15.1 & 16.4 & 16.0 & 21.0 & 20.2 \\
9 & 6.6 & 9.2 & 11.1 & 11.1 & 13.2 & 14.0 \\
10 & 6.9 & 11.3 & 12.3 & 8.0 & 12.7 & 17.0 \\
Missing & 0.0 & 0.7 & 0.4 & 0.5 & 1.1 & 0.4 \\
\hline
\end{tabular}

\section{References}

1. Jonkman, N. H., Schuurmans, M. J., Groenwold, R. H. H., Hoes, A. W., \& Trappenburg, J. C. A. (2016). Identifying components of self-management interventions that improve health-related quality of life in chronically ill patients: Systematic review and metaregression analysis. Patient Education and Counselling, 99(7), $1087-1098$.

2. Jonkman, N. H., Westland, H., Groenwold, R. H. H., Ågren, S., Anguita, M., Blue, L., Bruggink-André de la Porte P. W. F., DeWalt, D. A., Hebert, P. L., Heisler, M., Jaarsma, T., Kempen, G. I. J. M., Leventhal, M. E., Lok, D. J. A., Mårtensson, J., Muñiz, J., Peters-Klimm, O. H., Rich, F., Riegel, M. W., Strömberg, B., Tsuyuki, A., Trappenburg, R. T., Schuurmans, J. C. A., Hoes, M. J. (2016). What are effective program characteristics of self-management interventions in patients with heart failure? An individual patient data meta-analysis. Journal of Cardiac Failure, 22(11), 861-871.

3. Newham, J. J., Presseau, J., Heslop-Marshall, K., Russell, S., Ogunbayo, O. J., Netts, P., Hanratty, B., \& Kaner, E. (2017). Features of self-management interventions for people with COPD associated with improved health-related quality of life and reduced emergency department visits: A systematic review and meta-analysis. International Journal of Chronic Obstructive Pulmonary Disease, 12, 1705-1720.

4. Ogunbayo, O. J., Russell, S., Newham, J. J., Heslop-Marshall, K., Netts, P., Hanratty, B., \& Kaner, E. (2017). Understanding the factors affecting self-management of COPD from the perspectives of healthcare practitioners: A qualitative study. NPJ Primary Care Respiritory Medicine, 27(1), 54.

5. Liddy, C., Blazkho, V., \& Mill, K. (2014). Challenges of self-management when living with multiple chronic conditions: Systematic review of the qualitative literature. Canadian Family Physician, 60(12), 1123-1133.

6. Boger, E. J., Demain, S., \& Latter, S. (2013). Self-management: a systematic review of outcome measures adopted in self-management interventions for stroke. Disability and Rehabilitation, 35(17), 1415-1428.

7. Nolte, S., Elsworth, G. R., Newman, S., \& Osborne, R. H. (2013). Measurement issues in the evaluation of chronic disease self-management programs. Quality of Life Research, 22(7), 1655-1664.

8. Packer, T. L., Fracini, A., Audulv, Å, Alizadeh, N., van Gaal, B. G. I., Warner, G., \& Kephart, G. (2018). What we know about the purpose, theoretical foundation, scope and dimensionality of existing self-management measurement tools: A scoping review. Patient Education and Counselling, 101(4), 579-595.

9. Corrigan, J. M., Adams, K., \& Greiner, A. C. (2004). 1st annual crossing the quality chasm summit: A focus on communities. Washington, DC: National Academies Press.

10. Audulv, ̊, Packer, T., Hutchinson, S., Roger, K. S., \& Kephart, G. (2016). Coping, adapting or self-managing-What is the difference? A concept review based on the neurological literature. Journal of Advanced Nursing, 72(11), 2629-2643.

11. Hibbard, J. H., Stockard, J., Mahoney, E. R., \& Tusler, M. (2004). Development of the patient activation measure (PAM): Conceptualizing and measuring activation in patients and consumers. Health Services Research, 39(4 Pt 1), 1005-1026.

12. Hibbard, J. H., Mahoney, E. R., Stockard, J., \& Tusler, M. (2005). Development and testing of a short form of the patient activation measure. Health Services Research, 40 (6 Pt 1), 1918-1930.

13. Battersby, M. W., Ask, A., Reece, M. M., Markwick, M. J., \& Collins, J. P. (2003). The Partners in Health scale: The development and psychometric properties of a generic assessment scale for chronic condition self-management. Australian Journal of Primary Health, 9(3), 41-52. 
14. Smith, D., Harvey, P., Lawn, S., Harris, M., \& Battersby, M. (2017). Measuring chronic condition self-management in an Australian community: Factor structure of the revised Partners in Health (PIH) scale. Quality of Life Research, 26(1), 149-159.

15. Lorig, K., Chastain, R. L., Ung, E., Shoor, S., \& Holman, H. R. (1989). Development and evaluation of a scale to measure perceived self-efficacy in people with arthritis. Arthritis and Rheumatism, 32(1), 37-44.

16. Bandura, A. (1977). Self-efficacy: Toward a unifying theory of behavioral change. Psychological Review, 84(2), 191.

17. Bodenheimer, T., Wagner, E. H., \& Grumbach, K. (2002). Improving primary care for patients with chronic illness: the chronic care model, Part 2. JAMA, 288(15), 1909-1914.

18. Bodenheimer, T., Lorig, K., Holman, H., \& Grumbach, K. (2002). Patient self-management of chronic disease in primary care. JAMA, 288(19), 2469-2475.

19. Von Korff, M., Gruman, J., Schaefer, J., Curry, S. J., \& Wagner, E. H. (1997). Collaborative management of chronic illness. Annals of Internal Medicine, 127(12), 1097-1102.

20. Versnel, J., Packer, T., Weeks, L. E., Brown, J., Godwin, M., Hutchinson, S., Kephart, G., MacKenzie, D., Roger, K., Stadnyk, R., Villeneuve, M., \& Warner, G. (2013). The everyday experience of living with and managing a neurological condition (the LINC study): Study design. BMC Neurology, 13, 30.

21. Audulv, A., Packer, T., \& Versnel, J. (2014). Identifying gaps in knowledge: A map of the qualitative literature concerning life with a neurological condition. Chronic Illness, 10(3), 192-243.

22. Canada, P. H. A. O. (2014). Mapping Connections: An understanding of the neurological conditions in Canada. Ottawa: Government of Canada.

23. Raghunathan, T. E., Lepkowski, J. M., \& Hoewyk, J. V. (2001). A multivariate technique for multiply imputing missing values using a sequence of regression models. Survey Methodology, 27(1), 85-96.

24. StataCorp. (2017). Stata Statistical Software: Release 15. College Station: StataCorp LP.

25. Ritter, P. L., \& Lorig, K. (2014). The English and Spanish SelfEfficacy to Manage Chronic Disease Scale measures were validated using multiple studies. Journal of Clinical Epidemiology, 67(11), 1265-1273.

26. Freund, T., Gensichen, J., Goetz, K., Szecsenyi, J., \& Mahler, C. (2013). Evaluating self-efficacy for managing chronic disease: Psychometric properties of the six-item Self-Efficacy Scale in Germany. Journal of Evaluation in Clinical Practice, 19(1), 39-43.

27. Eslami, A., Daniali, S. S., Mohammadi, K., Reisi-Dehkordi, N., \& Mostafavi-Darani, F. (2017). Cultural adaptation and psychometric properties of the persian version of self-efficacy in chronic disease patients. Iranian Journal of Nursing and Midwifery Research, 22(1), 57-61.

28. Petkov, J., Harvey, P., \& Battersby, M. (2010). The internal consistency and construct validity of the partners in health scale: Validation of a patient rated chronic condition self-management measure. Quality of Life Research, 19(7), 1079-1085.

29. Brenk-Franz, K., Hibbard, J. H., Herrmann, W. J., Freund, T., Szecsenyi, J., Djalali, S., Steurer-Stey, C., Sönnichsen, A., Tiesler, F., Storch, M., Schneider, N., \& Gensichen, J. (2013). Validation of the German version of the patient activation measure 13 (PAM13D) in an international multicentre study of primary care patients. PLOS ONE, 8(9), e74786.

30. Graffigna, G., Barello, S., Bonanomi, A., Lozza, E., \& Hibbard, J. (2015). Measuring patient activation in Italy: Translation, adaptation and validation of the Italian version of the patient activation measure 13 (PAM13-I). BMC Medical Informatics and Decision Making, 15, 109.
31. Magnezi, R., \& Glasser, S. (2014). Psychometric properties of the Hebrew translation of the patient activation measure (PAM-13). PLoS ONE, 9(11), e113391.

32. Moreno-Chico, C., González-de Paz, L., Monforte-Royo, C., Arrighi, E., Navarro-Rubio, M. D., \& Gallart Fernández-Puebla, A. (2017). Adaptation to European Spanish and psychometric properties of the patient activation measure 13 in patients with chronic diseases. Family Practice, 34(5), 627-634.

33. Rademakers, J., Nijman, J., van der Hoek, L., Heijmans, M., \& Rijken, M. (2012). Measuring patient activation in The Netherlands: translation and validation of the American short form patient activation measure (PAM13). BMC Public Health, 12, 577.

34. Zill, J. M., Dwinger, S., Kriston, L., Rohenkohl, A., Härter, M., \& Dirmaier, J. (2013). Psychometric evaluation of the German version of the patient activation measure (PAM13). BMC Public Health, 13, 1027.

35. Moljord, I. E. O., Lara-Cabrera, M. L., Perestelo-Pérez, L., Rivero-Santana, A., Eriksen, L., \& Linaker, O. M. (2015). Psychometric properties of the patient activation measure-13 among outpatients waiting for mental health treatment: A validation study in Norway. Patient Education and Counselling, 98(11), 1410-1417.

36. Green, C. A., Perrin, N. A., Polen, M. R., Leo, M. C., Hibbard, J. H., \& Tusler, M. (2010). Development of the patient activation measure for mental health. Administration and Policy in Mental Health, 37(4), 327-333.

37. Skolasky, R. L., Mackenzie, E. J., Riley, L. H., \& Wegener, S. T. (2009). Psychometric properties of the patient activation measure among individuals presenting for elective lumbar spine surgery. Quality of Life Research, 18(10), 1357-1366.

38. Prey, J. E., Qian, M., Restaino, S., Hibbard, J., Bakken, S., Schnall, R., Rothenberg, G., Vawdrey, D. K., \& Masterson Creber, R. (2016). Reliability and validity of the patient activation measure in hospitalized patients. Patient Education and Counselling, 99(12), 2026-2033.

39. Hung, M., Carter, M., Hayden, C., Dzierzon, R., Morales, J., Snow, L., Butler, J., Bateman, K., \& Samore, M. (2013). Psychometric assessment of the patient activation measure short form (PAM-13) in rural settings. Quality of Life Research, 22(3), 521-529.

40. Packer, T. L., Kephart, G., Ghahari, S., Audulv, Å, Versnel, J., \& Warner, G. (2015). The patient activation measure: A validation study in a neurological population. Quality of Life Research, 24(7), 1587-1596.

41. Brown, T. A. (2015). Confirmatory factor analysis for applied research. New York: Guilford.

42. McNeish, D. (2017). Thanks coefficient alpha, we'll take It from here. Psychological Methods, 23(3), 412-433.

43. Sijtsma, K. (2009). On the use, the misuse, and the very limited usefulness of Cronbach's Alpha. Psychometrika, 74(1), 107-120.

44. Satorra, A.. Bentler, P. M. (1994). Corrections to test statistics and standard errors in covariance structure analysis. In von Eye A. \& C. C. Clogg (Eds.), In Latent variables analysis: Applications for developmental research (pp. 399-419). Thousand Oaks: Sage

45. Olsson, U. H., Foss, T., Troye, S. V., \& Howell, R. D. (2000). The performance of ML, GLS, and WLS estimation in structural equation modeling under conditions of misspecification and nonnormality. Structural Equation Modeling, 7(4), 557-595.

46. Hu, L.. Bentler, P. M. (1999). Cutoff criteria for fit indexes in covariance structure analysis: Conventional criteria versus new alternatives. Structural Equation Modeling: A Multidisciplinary Journal, 6(1), 1-55.

47. Ngooi, B. X., Packer, T. L., Kephart, G., Warner, G., Koh, K. W. L., Wong, R. C. C., \& Lim, S. P. (2016). Validation of the patient activation measure (PAM-13) among adults with cardiac conditions in Singapore. Quality of Life Research, 26(4), 1071-1080. 
48. Lenferink, A., Effing, T., Harvey, P., Battersby, M., Frith, P., van Beurden, W., et al. (2016). Construct validity of the Dutch version of the 12-item partners in health Scale: Measuring patient selfmanagement behaviour and knowledge in patients with chronic obstructive pulmonary disease. PLOS ONE, 11(8), e0161595.

49. Corbin, J.. Strauss, A. (1985). Managing chronic illness at home: Three lines of work. Qualitative Sociology, 8(3), 224-247.

50. Corbin, J. M., \& Strauss, A. (1988). Unending work and care: Managing chronic illness at home. San Fransisco, CA: Jossey-Bass.

51. Audulv, Å., Asplund, K., \& Norbergh, K.-G. (2012). The integration of chronic illness self-management. Qualitative Health Research, 22(3), 332-345.

52. Audulv, A., Norbergh, K. G., Asplund, K., Hörnsten Å. (2009). An ongoing process of inner negotiation-A grounded Theory study of self-management among people living with chronic illness. Journal of Nursing and Healthcare of Chronic Illness, 1(4), 283-293.

53. Schulman-Green, D., Jaser, S. S., Park, C., \& Whittemore, R. (2016). A metasynthesis of factors affecting self-management of chronic illness. Journal of Advanced Nursing, 72(7), 1469-1489.

54. Jamieson, N. J., Hanson, C. S., Josephson, M. A., Gordon, E. J., Craig, J. C., Halleck, F., Budde, K., \& Tong, A. (2016). Motivations, challenges, and attitudes to self-management in kidney transplant recipients: A systematic review of qualitative studies. American Journal of Kidney Diseases, 67(3), 461-478.
55. Boehmer, K. R., Gionfriddo, M. R., Rodriguez-Gutierrez, R., Dabrh, A. M., Leppin, A. L., Hargraves, I., May, C. R., Shippee, N. D., Castaneda-Guarderas, A., Palacios, C. Z., Bora, P., Erwin, P., \& Montori, V. M. (2016). Patient capacity and constraints in the experience of chronic disease: A qualitative systematic review and thematic synthesis. BMC Family Practice, 17, 127.

56. Roberts, N. J., Kidd, L., Dougall, N., Patel, I. S., McNarry, S., \& Nixon, C. (2016). Measuring patient activation: The utility of the patient activation measure within a UK context-results from four exemplar studies and potential future applications. Patient Education and Counselling, 99(10), 1739-1746.

57. Skolasky, R. L., Green, A. F., Scharfstein, D., Boult, C., Reider, L., \& Wegener, S. T. (2011). Psychometric properties of the patient activation measure among multimorbid older adults. Health Services Research, 46(2), 457-478.

58. Mosen, D. M., Schmittdiel, J., Hibbard, J., Sobel, D., Remmers, C., \& Bellows, J. (2007). Is patient activation associated with outcomes of care for adults with chronic conditions? Journal of Ambulatory Care Management, 30(1), 21-29.

59. Smith, D., Lawn, S., Harvey, P., \& Battersby, M. (2017). Concurrent validity of the Partners in Health scale against general selfrated health in chronic conditions: A short report. Chronic Illness. https://doi.org/10.1177/1742395317743559. 